\title{
Successful Clinical Management of Temporary Blindness In A Horse: A Case Report
}

\author{
Rekha Pathak ${ }^{1}$ and Pankaj ${ }^{2}$ \\ ${ }_{1}^{1}$ senior scientist, Division of Surgery, \\ Indian Veterinary Research Institute, Izatnagar, UP -243122, India \\ ${ }^{2}$ Veterinary Officer, Kichha,UP. India
}

\begin{abstract}
Abstrac: Treatment of progressive blindness of both the eyes was attempted in field condition in horse. In this study, a case of equine was presented and since there was constraint of proper diagnosis and treatment, how the case was successfully managed in field condition was projected. After a careful examination, the treatment protocol included Injections of streptopencillin $2.5 \mathrm{~g}$ daily twice intramuscularly along with multivitamin injection Hivit daily once for one week and Inj. Melonex was given $10 \mathrm{ml}$ intramuscularly daily for one week was given. The topical and systemic anti-inflammatory medications i.e. Genticyn- HC drops locally and betnesol eye ointment $0.1 \% \mathrm{w} / \mathrm{w}$ (Glaxo) was used $2-3$ times a day. With this we could successfully treat the case within a week period.
\end{abstract}

Keyword: Leopard; Immobilization; Xylazine-ketamine; Atepamezole

\section{Introduction:}

Uveitis is an immune-mediated disease. "Impairment of the normal blood-aqueous barrier in the iris and ciliary body vasculature owing to inflammation is the underlying cause of the clinical signs (Kern, 1987). Uveitis unfortunately is often misdiagnosed as something less severe or in some cases it is totally ignored. It is important that uveitis be diagnosed correctly because lack of or incorrect treatment can have serious consequences (Nelson, 1995).

Case presentation: A male horse of age six yrs was brought to veterinary hospital Deorania District Bareilly, with the history of development of progressive blindness of both the eyes. There was no associated history of trauma or accident. Rectal temperature was found to be $103^{0} \mathrm{~F}$ and the heart rate was 45 per minute. It was reported that the horse was not taking feed properly for the past one week. Due to the limited facilities available in the field only the animal's faecal examination could be done which was found to be negative for any parasitic ova.

\section{Clinical examination and treatment}

On examination of eye carefully it was found that animal had blepherospasms, epiphora, corneal edema, and fibrin clots in the anterior chamber with miosis. Which are the typical signs of acute uveitis. The animal was carefully examined for the reflexes. It was found that animal was having his bilateral vision compromised. The pupillary reflex was found to be present but the animal was unable to walk straight due to blindness. Injections of streptopencillin $2.5 \mathrm{~g}$ daily twice was administered intramuscularly along with multivitamin injection Hivit daily once for one week and Inj. melonex was given $10 \mathrm{ml}$ intramuscularly daily for one week.

Although diagnosis of the causative agent in this case could not be made due to inadequate facilities but the fact that acute uveitis may be caused due to leptospira infection, which is one of the most common ocular diseases in horses characterized by episodes of active inflammation followed by varying periods of quiescence led us to take a decision on treating the case symptomatically.

The topical and systemic anti-inflammatory medications i.e. Genticyn- HC drops locally and betnesol eye ointment $0.1 \% \mathrm{w} / \mathrm{w}$ (Glaxo) was used 2-3 times a day. Injections of meloxicam $15 \mathrm{ml}$ intravenously was given for one week had solved the problem of pain and inflammation and also the corneal edema.

Atropine ointment was also used locally which paralyses the iris sphincter and cilliary body musculature. These effects reduce the muscle spasms but along with it a regular history of the defecation was taken to monitor the gut motility which is affected by atropine applied topically also.

Injection dexamethasone $10 \mathrm{ml}(4 \mathrm{mg} / \mathrm{ml})$ was also given initially for a period of four days intramuscularly. Amazingly it was found that the animal slowly recovered from the compromised vision and started to have a near normal vision within a week period. The treatment was continued for a period of one more week after a gap of another four days. 


\section{Discussion}

we initiated the treatment at the earliest possible. Equine recurrent uveitis is one of the most common ocular diseases in horses, classically characterized by episodes of active inflammation followed by varying periods of quiescence. During the so-called quiescent periods, low-grade, subclinical inflammation may continue in at least some individuals. Regardless of the specific course, the inflammatory events eventually lead to secondary changes. It is these adverse secondary complications that make this syndrome the most common cause of blindness in horses worldwide. The clinical signs associated with equine recurrent uveitis include both acute signs of active inflammation and chronic secondary side effects. Damage to the uveal tract leads to the release of inflammatory mediators such as leukotrienes, prostaglandins, and histamines, which in turn causes increased permeability of anterior uveal vessels, breakdown of the blood-aqueous barrier, iris sphincter spasm, and ciliary body muscle spasm. The compromise of the blood-aqueous barrier allows for leakage of protein, fibrin, and cells into the aqueous (Merk veterinary Manual, 2005). In this study we used topical betamethasone eye ointment $(0.1 \%)$ for the instant relief. Both steroidal and nonsteroidal topical medications are commonly used. Prednisolone acetate (steroid, $1 \%$ suspension), dexamethasone (steroid, $0.1 \%$ suspension or ointment), flurbiprofen (nonsteroidal, $0.03 \%$ solution), and diclofenac (nonsteroidal, $0.1 \%$ solution) have all been successfully used. In this case study we used streptopencillin to treat the infection. Signs of leptospirosis include fever, anorexia, and conjunctivitis in domestic livestock. Streptomycin and penicillin are used to treat leptospirosis (Pinney, 1986).

The present study was presented in the field conditions and due to lack of facilities all the necessary examinations could not be made. We still attempted a treatment which was aimed to provide a symptomatic relief to the animal and it was successfully done.

\section{Conflict of interest}

Authors have no conflict of interest

\section{References}

[1]. Kern T.J. (1987). Intraocular inflammation, in robinson, n.e.(ed): current therapy in equine medicine medicine-2, philadelphia, w. b. saunders company, 445-450.

[2]. Nelson M.G. (1995). Equine recurrent uveitis a survey of 68 horses in the United States and Canada.

[3]. Pinney Chris C. (1986). Leptospirosis: prevention/control in domestic livestock, The southwestern veterinarian. 37(1): 51-55.

[4]. Radostits O.M., Blood D.C., Gay O.C. (1994). A textbook of the Diseases of Cattle, Sheep, Pig, Goat and Horses. ELBS 8th EDN

[5]. The Merk Veterinary Manual (9th Edn.) 2005, Printed by National Publishing, Inc. Philadelpia, Pennsylvania 